\title{
Optimizing appointment template and number of staff of an OB/GYN clinic - micro and macro simulation analyses
}

\author{
R.B. Lenin ${ }^{1}$, Curtis L. Lowery², Wilbur C. Hitt ${ }^{2}$, Nirvana A. Manning ${ }^{2}$, Peter Lowery ${ }^{3}$ and Hari Eswaran²*
}

\begin{abstract}
Background: The Department of Obstetrics and Gynecology (OB/GYN) at the University of Arkansas for Medical Sciences (UAMS) tested various, new system-restructuring ideas such as varying number of different types of nurses to reduce patient wait times for its outpatient clinic, often with little or no effect on waiting time. Witnessing little progress despite these time-intensive interventions, we sought an alternative way to intervene the clinic without affecting the normal clinic operations.

Aim: The aim is to identify the optimal (1) time duration between appointments and (2) number of nurses to reduce wait time of patients in the clinic.

Methods: We developed a discrete-event computer simulation model for the OB/GYN clinic. By using the patient tracker (PT) data, appropriate probability distributions of service times of staff were fitted to model different variability in staff service times. These distributions were used to fine-tune the simulation model. We then validated the model by comparing the simulated wait times with the actual wait times calculated from the PT data. The validated model was then used to carry out "what-if" analyses.
\end{abstract}

Results: The best scenario yielded 16 min between morning appointments, 19 min between afternoon appointments, and addition of one medical assistant. Besides removing all peak wait times and bottlenecks around noon and late in the afternoon, the best scenario yielded $39.84 \%(p<.001), 30.31 \%(p<.001)$, and $15.12 \%$ $(p<.001)$ improvement in patients' average wait times for providers in the exam rooms, average total wait time at various locations and average total spent time in the clinic, respectively. This is achieved without any compromise in the utilization of the staff and in serving all patients by $5 \mathrm{pm}$.

Conclusions: A discrete-event simulation model is developed, validated, and used to carry out "what-if" scenarios to identify the optimal time between appointments and number of nurses. Using the model, we achieved a significant improvement in wait time of patients in the clinic, which the clinic management initially had difficulty achieving through manual interventions. The model provides a tool for the clinic management to test new ideas to improve the performance of other UAMS OB/GYN clinicS.

Keywords: Outpatients, Patient types, Patient tracker data, Appointment template, Exam rooms, Optimization, Utilization, Simulation

\footnotetext{
*Correspondence: EswaranHari@uams.edu

2 Department of OB/GYN, University of Arkansas for Medical Sciences, 4301

West Markham Street, 72205 Little Rock, Arkansas, USA

Full list of author information is available at the end of the article
} 


\section{Background}

Healthcare delivery is becoming increasingly complicated and difficult to provide efficiently. Patient satisfaction is a powerful force in the health care industry and is being measured by governing institutions to improve healthcare delivery. High levels of patient satisfaction are associated with better outcomes, and waiting time is directly related to patient dissatisfaction. In an ideal world, no patient would ever wait to see his or her doctor, thus erasing the necessity of a waiting room. However, in today's ever increasingly busy medical clinic, waiting time is an unavoidable reality, so identifying ways to lessen wait time would be ideal, considering the many negative consequences of increased wait time [1,2]. Anecdotally speaking, the majority of patients' negative feedback includes comments on wait time [3, 4]. Studies too have shown long wait times are associated with low patient satisfaction scores $[5,6]$. It has been established that patients who are satisfied with their care are more likely to adhere to treatment [7]. Low patient satisfaction affects treatment compliance, including return visit rates $[1,8]$.

\section{Available tools}

Many medical practices use a "trial and error" method to determine the optimal clinical arrangement, which often results in failed attempts and settling for a "good enough" option. Operations research techniques such as queuing theory, optimization techniques, and discrete-event simulations have been developed to understand and improve outpatient clinic performance [9-11]. Mathematical models based on queuing theory and linear and non-linear programming techniques often suffer from unrealistic assumptions which oversimplify the actual system [12].

\section{Simulation models}

Discrete-event simulation models have been used in modeling complex outpatient clinic situations because they can depict the actual clinic without unrealistic assumptions while also accurately capturing random variations of the clinic [13]. Simulation models have been used successfully for outpatient clinics to streamline patient flows and to optimize resource allocations and utilization $[14,15]$. In healthcare systems, simulation models can explore various performance measures such as patients' wait time, resource utilization, resource allocation, system capacity, and appointment scheduling without altering the actual system [10, 16]. As opposed to continuous simulation, discrete-event simulation (DES) can test systems that change states at discrete epochs over time [17]. In this paper, we collected patient wait times at time epochs when they moved from one location to another location within the clinic. Since these movements take place at discrete time points, DES is a natural choice to our work.

\section{Appointment scheduling system}

Outpatient clinics are often driven by appointment scheduling systems that infrequently accommodate sameday appointments. It is critical to have efficient appointment schedules in order to deliver high quality of service [18]. Several methods have been proposed to design appointment schedules [18-21]. Researchers tested the idea of scheduling multiple patients per block and found that it reduced patient wait time and doctors' idle time in some cases and increased in some other cases $[22,23]$. The variability in service times of providers, nature of treatment, and type of patients affect the performance of appointment systems [24]. For this reason, any generalized appointment scheduling rule will not necessarily improve the performance of a clinic $[21,25,26]$.

\section{Resource allocation and utilization}

Rising costs make it difficult for the health care services to recruit more staff. Hence, identifying optimum number of staff is pertinent to match the demand while maximizing staff utilization and minimizing patients' wait time. The healthcare community has used simulation models effectively to identify optimal number of resources [27, 28]. Studying the changes to clinic resources and appointment scheduling policy simultaneously was found to be very effective in reducing patient wait times without compromising the resources' utilization $[29,30]$.

\section{Simulation and optimization techniques}

In order to automate the process of identifying optimal values for the simulation parameters such as the number of resources and time between appointments, suitable and efficient optimization techniques are needed to carry out several "what-if" scenarios with varying parameter values. Combination of optimization techniques and simulation techniques is found to be useful and efficient in simulating healthcare systems [31-33].

\section{The problem and proposed solution}

The University of Arkansas for Medical Sciences (UAMS) recently opened a women's health and internal medicine outpatient clinic in a high-growth area of Little Rock, Arkansas, with the mission to recruit and serve residents of this area through this conveniently located clinic, referred as the West Little Rock (WLR) clinic. To encourage patient retention at the new clinic, UAMS placed a high priority on decreasing patient wait times in the clinic. The new clinic very soon started facing long patient wait time around noon and late in the afternoon. To tackle this problem, the UAMS OB/GYN administration had tested various, new system-restructuring ideas such as changing the appointment scheduling system and the number of nurses, often with little or no success. 
Witnessing little progress despite these time-intensive interventions, we proposed to develop a simulation model of the clinic to test new ideas to reduce patients' wait time. By using the patient tracker (PT) data to fit probability distributions of service times of staff, the simulation model was fine-tuned further to test proposed changes to the system. We then validated the model by comparing the simulated wait times with the actual wait times calculated from the PT data.

In this work, we combined the validated simulation model and optimization technique to carry out "what-if" scenarios to identify an optimal appointment system and an optimal number of nurses. The results of these scenarios are discussed in detail through tables and time series graphs.

To further the discussion, the proceeding sections explore the layout and characteristics of the study site, the data collection process, the simulation software and study-specific development, the results, discussions, conclusions, and future research plan.

\section{Methods \\ Clinic description \\ Patients}

The WLR clinic handles three classes of patients: normal obstetric care $(\mathrm{OB})$, complete gynecological service (GYN), and internal medicine service (IM). In addition, a fourth class of OB patients is handled by the clinic who had only ultrasound (US) appointments. Each of the first three classes involves two types of patients: new (NOB, NGYN, and NIM) and return (ROB, RGYN, and RIM) patients. Since the UAMS OB/GYN department elected to carry out "what-if" scenarios, this paper focuses only on the OB and GYN patient classes. However, all patients of the clinic share the common lab for blood work; therefore, the IM clinic is implemented in the simulation model and its details are omitted in this work. A typical flow of $\mathrm{OB} / \mathrm{GYN}$ patients in the clinic is shown in Fig. 1.

\section{Clinic layout}

Figure 2 shows the layout of the WLR clinic. The left side of the clinic is dedicated to OB/GYN patients and services, while the right side of the clinic serves IM patients. The front desk (225), waiting room (201), and one single capacity lab room (222) are the only locations in the clinic used by both OB/GYN and IM patients. In addition to these locations, the OB/GYN clinic has seven single capacity exam rooms (205 - 209, 213, and 217), one doctors' office (214), one single capacity US room (218), and one nurses' station (219).

\section{Types of staff}

There are five OB/GYN doctors, and they are referred to as Dr. A through Dr. E. There is one registered nurse (RN), two licensed practical nurses (LPNs), one medical assistant (MA), one patient representative (PR), two access coordinators (ACs), and one ultrasound technician (UST). The RN escorts patients from waiting room to exam rooms, checks vital signs, and chaperones GYN patients in the exam room if needed by the doctors. The duties of the two LPNs are the same as the RN. The MA conducts lab work for both OB/GYN and IM clinics. If available, she also chaperones GYN patients in the exam rooms and escorts patients from waiting room to exam room, but either an RN or LPN checks the vital signs of these patients. The PR assists with check-in when needed, balances the cash collection/batching, counsels patients on financial aspects, and also obtains all pre-authorizations for both clinics. The ACs manage the front desk: one for check-in and another for check-out. The UST performs diagnostic medical sonographic procedures. The working schedules of staff are tabulated in Table 1. On any working day, there are two physicians scheduled except on Wednesday when two 0.5 physicians (each worked a half day) are scheduled.

\section{Appointment (scheduling) template of providers}

In total, 16 patients in the morning and 12 patients in the afternoon are scheduled for each provider. Out of 28, five and two are new patients scheduled in the morning and afternoon sessions, respectively, as per the clinic director's recommendation. Hence, 56 patients are scheduled on Monday, Tuesday, Thursday, and Friday, and

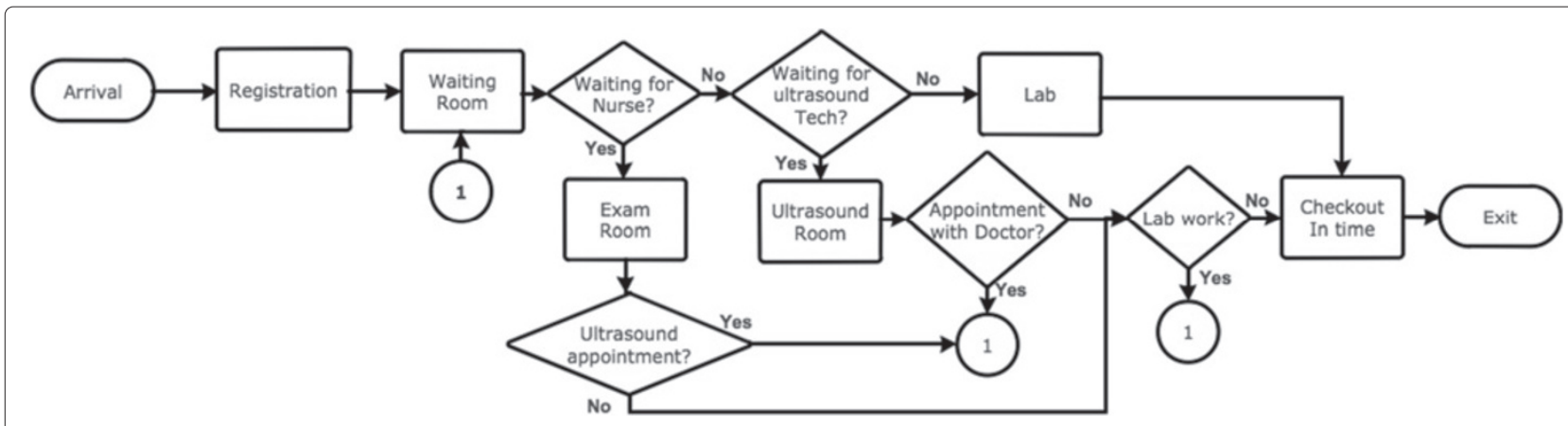

Fig. 1 Flow of OB/GYN patients in the clinic 


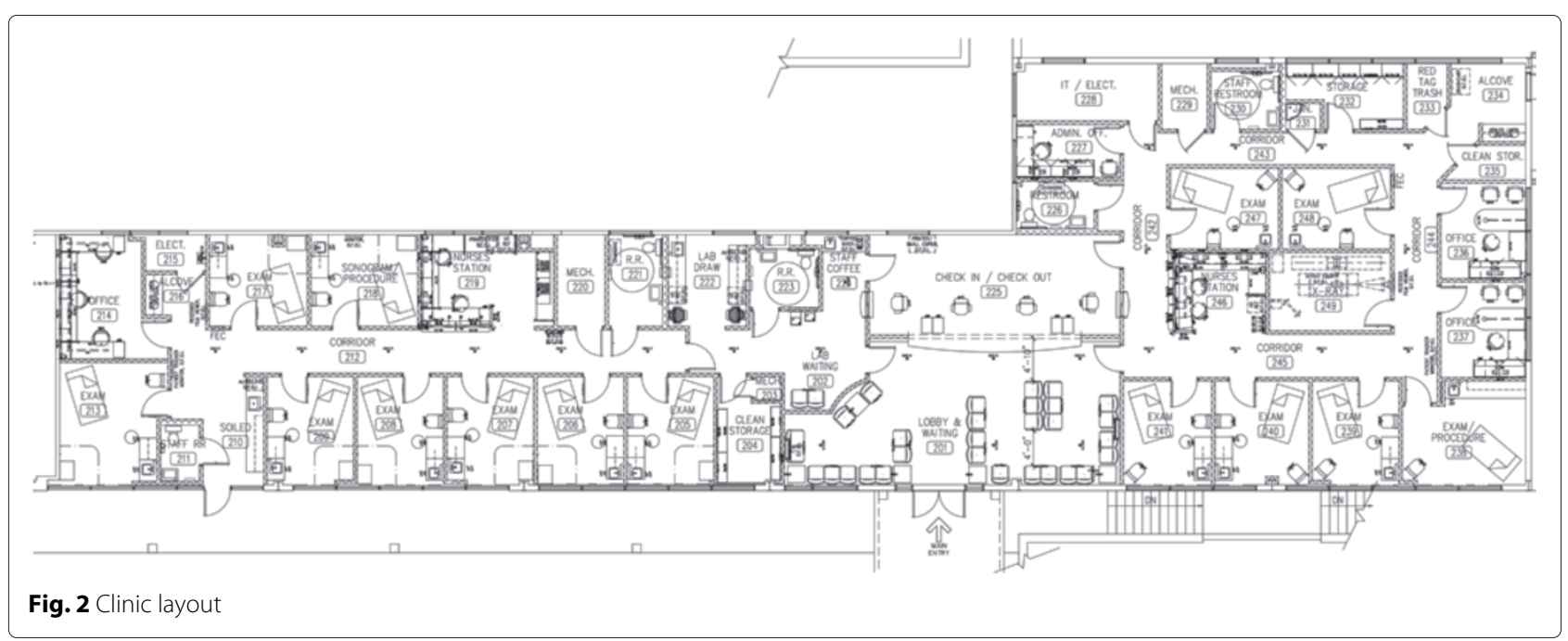

24 patients on Wednesday. The appointment scheduling template currently used for a provider is shown in Table 2. The appointment times denote the appointment times with the providers and not for the ultrasound (US) procedure. US appointments preceded the providers' scheduled appointments and they are not considered in this study. The performance measures $W T, T W T$, and TST (refer Table 5 for details) do not include US procedures.

\section{Data}

\section{Patient tracker}

Patient tracker (PT) is an in-house built, visualization and touch-screen software installed on all computers at various locations of the clinic, such as the front desk, exam rooms, US room, and labs. It was originally intended to record available exam rooms and assign patients to those rooms from the waiting room. The software was modified with additional buttons to record timestamps (milestones)

Table 1 Working schedules of staff

\begin{tabular}{|c|c|c|c|c|c|}
\hline & Mon & Tue & Wed & Thu & Fri \\
\hline Dr. A & & $A M \& P M$ & & PM & $A M \& P M$ \\
\hline Dr. B & & & & $A M \& P M$ & $A M \& P M$ \\
\hline Dr. C & & $A M \& P M$ & PM & & \\
\hline Dr. D & $A M \& P M$ & & & AM & \\
\hline Dr. E & AM \& PM & & PM & & \\
\hline$P R$ & AM \& PM & AM \& PM & $A M \& P M$ & $A M \& P M$ & $A M \& P M$ \\
\hline $\mathrm{RN}$ & $A M \& P M$ & $A M \& P M$ & $A M \& P M$ & $A M \& P M$ & $A M \& P M$ \\
\hline LPN & AM \& PM & $A M \& P M$ & AM \& PM & $A M \& P M$ & $A M \& P M$ \\
\hline$M A$ & $A M \& P M$ & $A M \& P M$ & AM \& PM & $A M \& P M$ & $A M \& P M$ \\
\hline$A C$ & $A M \& P M$ & $A M \& P M$ & $A M \& P M$ & $A M \& P M$ & $A M \& P M$ \\
\hline UST & AM & AM & & AM & AM \\
\hline
\end{tabular}

AM denotes the morning session and PM denotes the afternoon session whenever the staff interacted with patients at various locations. The staff were trained to use these added buttons. The milestones that were captured by the software are shown in Fig. 3.

Six months of data were collected for this study. During this period, the timestamps of more than 300 patients were collected for each staff listed in Table 1. From the data, the following details were gathered: (1) Show up percentages of patients, (2) service times of staff (amount of time a staff spent with a patient), (3) wait time of patients at various locations, (4) total wait times of each patient in the clinic, and (5) total spent time of each patient in the clinic. The total wait time of a patient is calculated by

Table 2 Appointment scheduling template

\begin{tabular}{|c|c|c|c|}
\hline \multicolumn{2}{|c|}{ Morning Session } & \multicolumn{2}{|c|}{ Afternoon Session } \\
\hline Time & Procedure & Time & Procedure \\
\hline $7: 45$ & ROB \& NGYN & $12: 45$ & ROB \& NGYN \\
\hline 8:00 & RGYN & $13: 00$ & RGYN \\
\hline $8: 15$ & NOB \& ROB & $13: 15$ & NOB \& ROB \\
\hline $8: 30$ & RGYN & $13: 30$ & $R O B \&$ RGYN \\
\hline $8: 45$ & NGYN & $13: 45$ & $\mathrm{ROB}$ \\
\hline 9:00 & $\mathrm{ROB}$ & $14: 00$ & $\mathrm{ROB}$ \\
\hline $9: 15$ & NOB & $14: 15$ & $\mathrm{ROB}$ \\
\hline $9: 30$ & RGYN & $14: 30$ & SDA \\
\hline $9: 45$ & $\mathrm{ROB}$ & $14: 45$ & SDA \\
\hline $10: 00$ & RGYN & & \\
\hline $10: 15$ & $\mathrm{NOB}$ & & \\
\hline $10: 30$ & RGYN & & \\
\hline $10: 45$ & $\mathrm{ROB}$ & & \\
\hline $11: 00$ & $\mathrm{ROB}$ & & \\
\hline
\end{tabular}

SDA stands for Same Day Appointment: ROB or RGYN - $50 \%$ - $50 \%$ 


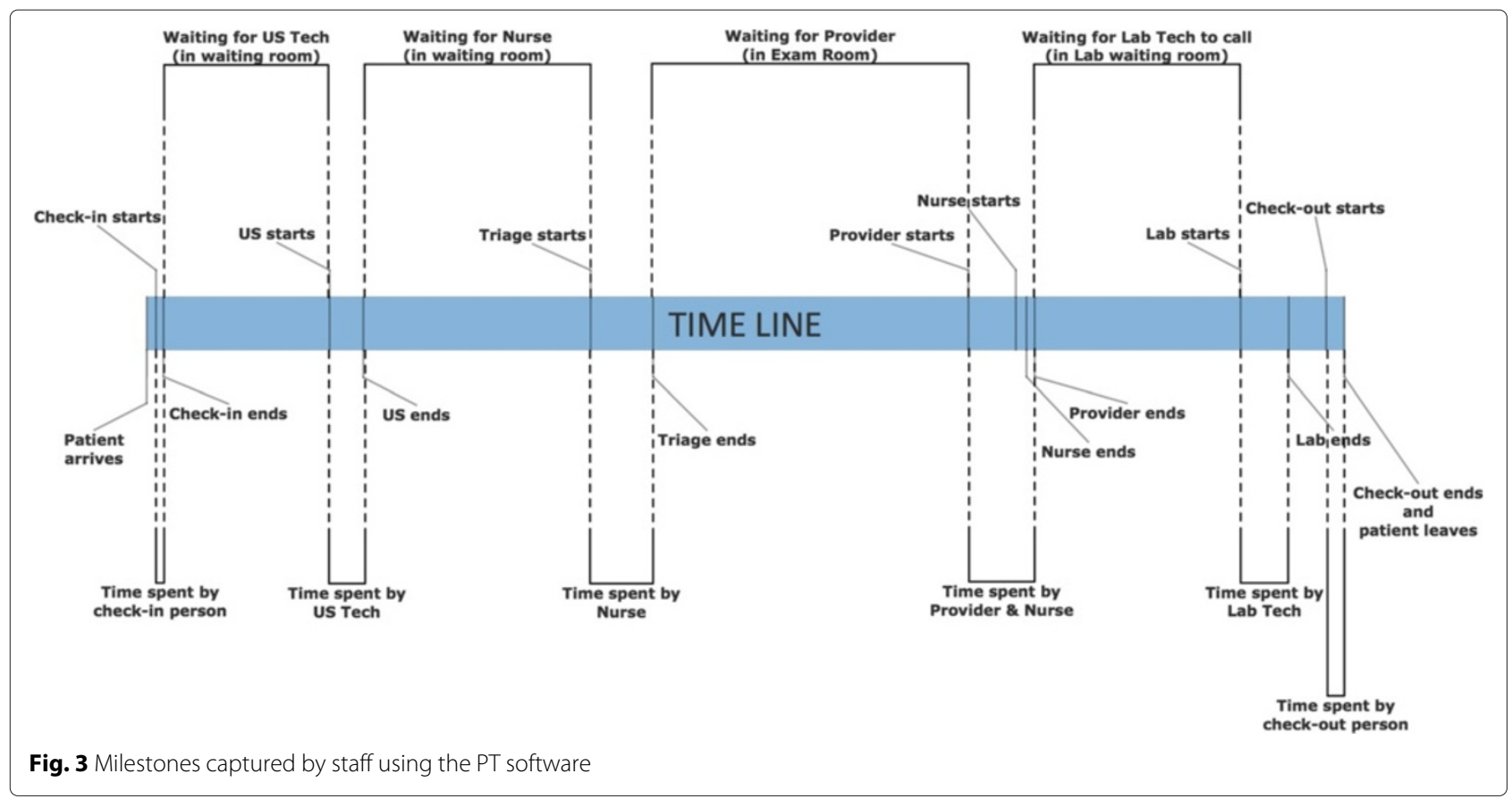

adding her wait times at various locations of the clinic. $T W T$ is the average of total wait times. The total spent time of a patient is calculated by finding the difference in her check-in and check-out times. TST is the average of total spent times. The first two information are used in building the simulation model, and the last three are used to validate the simulation model.

\section{Arrival times of patients}

During UST working days (refer Table 1), US appointments were scheduled for every $30 \mathrm{~min}$ starting from $8 \mathrm{am}$ to $11 \mathrm{am}$. US appointments preceded the providers' scheduled appointments. The arrival times of patients, who had ultrasound procedure appointments, denote the times they come back to the waiting room after ultrasound procedure. For patients who did not have ultrasound procedure appointment, their arrival times denote the times they arrived at the clinic. The distribution of time differences between appointment time with the provider and the arrival times of patients was found to be normally distributed with mean 0 min and standard deviation $(S D) 7 \mathrm{~min}$. In addition, new patients were instructed to come $15 \mathrm{~min}$ early to complete the paperwork. We used this information in the simulation model while generating patients according to the appointment template.

\section{Show up percentages}

Show up percentages of patients of the five OB/GYN doctors are tabulated in Table 3 . These percentages are the ratio of the actual number of arrived patients calculated from the PT data to the total number of scheduled patients calculated from the appointment template.

\section{Service times of staff}

Extracted service times (in minutes) of all staff from the PT data were used to identify suitable probability distributions using the Stat:Fit software [34]. Stat:Fit includes 32 probability distributions. Details about the notations and parameters of these distributions can be found in the user's manual of the software. Stat::Fit ranks the best fit according to $p$ values of two hypothesis tests: Kolmogorov-Smirnov (KS) and Anderson-Darling (AD).

Table 3 Show up percentages of patients

\begin{tabular}{lllllll}
\hline & & Mon & Tue & Wed & Thu & Fri \\
\hline Dr. A & New Pt (\%) & & 80 & & 70 & 90 \\
& Ret Pt (\%) & & 75 & & 75 & 78 \\
Dr. B & New Pt (\%) & & & & 71 & 75 \\
& Ret Pt (\%) & & & & 50 & 66 \\
Dr. C & New Pt (\%) & 90 & 100 & & \\
& Ret Pt (\%) & & 83 & 94 & & \\
*Dr. D & New Pt (\%) & 93 & & & 55 & \\
& Ret Pt (\%) & 80 & & & 100 & \\
Dr. E & New Pt (\%) & 56 & & & 100 & \\
& Ret Pt (\%) & 80 & & 95 & & \\
\hline
\end{tabular}


A screenshot of a fitted distributions is shown in Fig. 4. Though more than one distribution was identified by Stat::Fit as good fit for each staff member's service time, they produced statistically significantly the same simulation results. Thus, in the simulation model, we used the top-ranked fitted distributions that are tabulated in Table 4 along with $p$ values.

\section{Percentage of patients who had lab work}

From the PT data, we computed the percentages of patients who had lab work. They were $100 \%$ for NOB, $30 \%$ for ROB, 60 \% for NGYN, and $60 \%$ for RGYN. In addition, $100 \%$ of NIM and $30 \%$ of RIM had lab work. Since the lab is used by both OB/GYN and IM clinics, this information is used in the simulation model.

Ethics statement. This was a simulation and modeling quality improvement (QI) project and generic times spent in different locations in clinic were computed from an existing template and no identifiable patient specific information was used in any part of the project. It was determined that this does not meet the definition of human subject research that would be subject to UAMS IRB oversight. It is not required under IRB Policy 1.4. The authors can provide all the generic data incorporated in the project with no specific information other than data related to time spent in each location averaged over many entries be made available to the journal or other researchers on request within guidelines of the institution.

\section{Simulation model \\ MedModel}

We used MedModel [35], a discrete-event simulation software, to develop the simulation model for the clinic. The major steps to build a simulation in MedModel are sequenced logically: locations, entities (patients), resources (staff), arrivals, and processing. Most of the codings in processing steps define flows of patients through the clinic and how they are processed by the staff. Figure 5 is a screenshot of the model with process tables. Codings which are repeated in different processes for various parameters are modularized as subroutines. Figure 6 is a screenshot of the model with subroutines. MedModel's visualization feature helps the developer to validate the flow of patients and staff in the clinic. Figure 7 is a screenshot of a running simulation in MedModel. MedModel's scenario manager helps researchers carry out various "what-if" scenarios manually to identify optimal values for the clinic parameters.

\section{Simulation code}

Since there are more than 4000 lines of coding involved in developing the simulation model, we made these codes available in a simple text format at sites.google.com/a/uca. edu/rblenin/biomed.

\section{Input parameters}

We used the following information as input parameters for the simulation model: (1) Types and number of

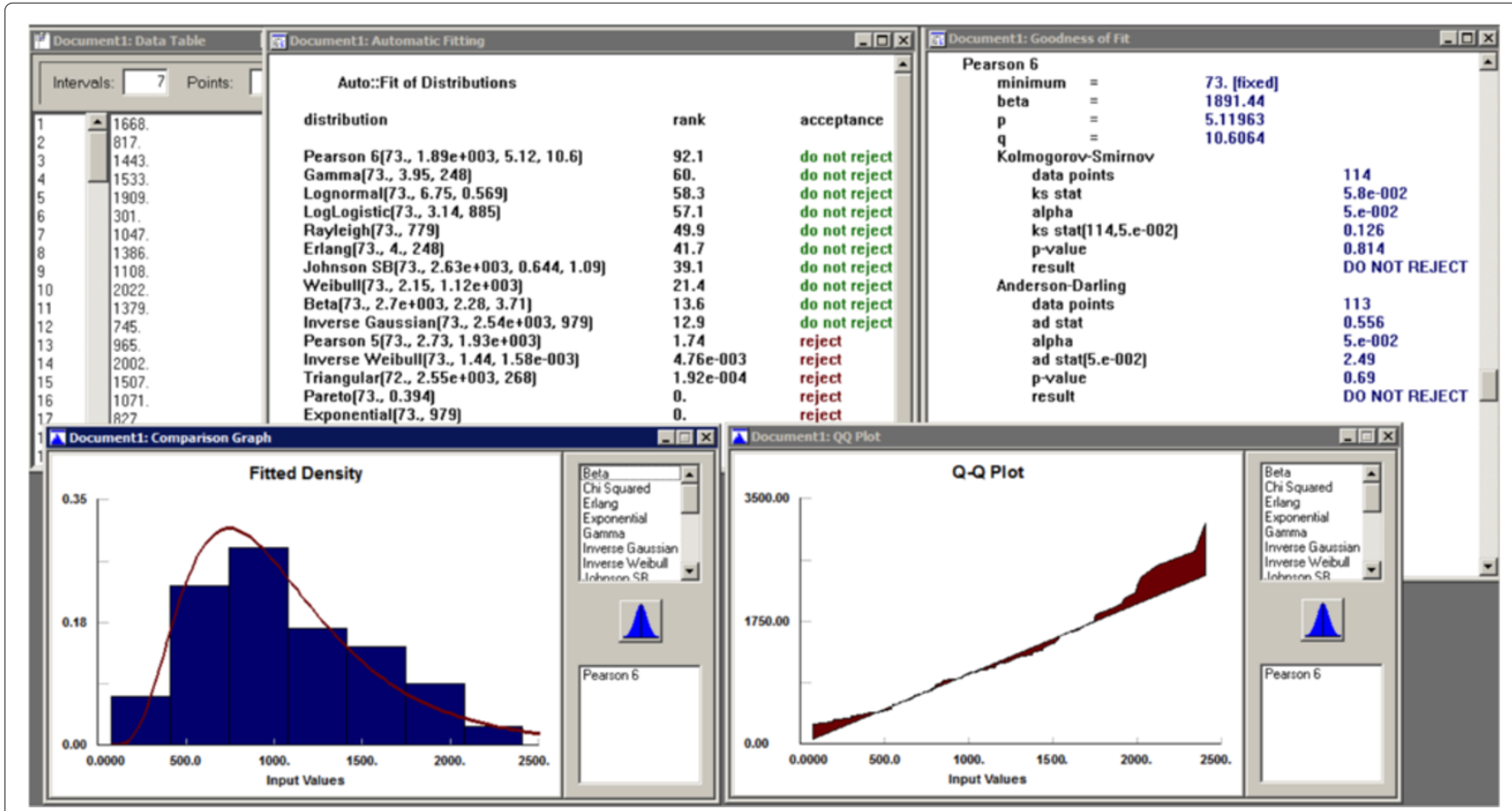

Fig. 4 Sample output of Stat::Fit 
Table 4 Fitted service time distributions of staff

\begin{tabular}{|c|c|c|c|c|}
\hline & & Fitted distribution & KS $p$ value & $\mathrm{AD} p$ value \\
\hline \multirow[t]{2}{*}{ US tech } & New Pt & Triangular $(10,15,20)$ & .427 & .318 \\
\hline & Ret Pt & Triangular $(10,15,20)$ & .631 & .434 \\
\hline \multirow[t]{2}{*}{ Check-in staff } & New Pt & Weibull $(5,1.96,700) / 60.0$ & 685 & 677 \\
\hline & Ret Pt & Beta $(5,2.1 \mathrm{e}+003,1.96,14.6) / 60.0$ & .884 & .751 \\
\hline \multirow[t]{2}{*}{ Nurse } & New Pt & LogLogistic $(100,2.92,417) / 60.0$ & .934 & .976 \\
\hline & Ret Pt & Pearson6 $(51,1.32 \mathrm{e}+003,2.31,9.93) / 60.0$ & .821 & .81 \\
\hline \multirow[t]{2}{*}{ Dr. A } & New Pt & Pearson6 $(73,1.89 e+003,5.12,10.6) / 60.0$ & .814 & 69 \\
\hline & Ret Pt & Pearson6 $(8,1.99 \mathrm{e}+003,3.14,9.16) / 60.0$ & .884 & .834 \\
\hline \multirow[t]{2}{*}{ Dr. B } & New Pt & Gamma $(30,3.02,391) / 60.0$ & .995 & .979 \\
\hline & Ret Pt & LogLogistic $(2,2.57,818) / 60.0$ & .996 & .913 \\
\hline \multirow[t]{2}{*}{ Dr. C } & New Pt & Weibull $(185,1.71,904) / 60.0$ & .998 & .999 \\
\hline & Ret Pt & LogLogistic $(36,2.85,555) / 60.0$ & .791 & 659 \\
\hline \multirow[t]{2}{*}{ Dr. D } & New Pt & Erlang $(61,5,188) / 60.0$ & .941 & .777 \\
\hline & Ret Pt & Gamma $(20,2.38,285) / 60.0$ & .52 & .513 \\
\hline \multirow[t]{2}{*}{ Dr. E } & New Pt & LogLogistic $(121,3.17,741) / 60.0$ & .967 & .944 \\
\hline & Ret Pt & Pearson6 $(22,461,8.31,6.62) / 60.0$ & 641 & 664 \\
\hline \multirow[t]{2}{*}{ Lab tech } & New Pt & Pearson6 $(1,4.95 e+003,1.57,18.5) / 60.0$ & .911 & .992 \\
\hline & Ret Pt & Lognormal $(9,5.87, .995) / 60.0$ & .221 & .172 \\
\hline \multirow[t]{2}{*}{ Check-out staff } & New Pt & Triangular $(1,2,3)$ & 628 & .547 \\
\hline & Ret Pt & Triangular $(1,2,3)$ & .846 & .652 \\
\hline
\end{tabular}

patients scheduled, (2) types and number of staff, (3) appointment templates of providers and UST, (4) show up percentages of patients, (5) arrival times of patients, (6) service times of staff, and (7) percentages of different types of patients who had lab work.

\section{Output parameters}

We measured the following output from the simulation model: (1) WT, (2) TWT, (3) TST, (4) utilization of staff and exam rooms, and (5) throughput. We then compared the measures (1) - (3) with the ones computed from the

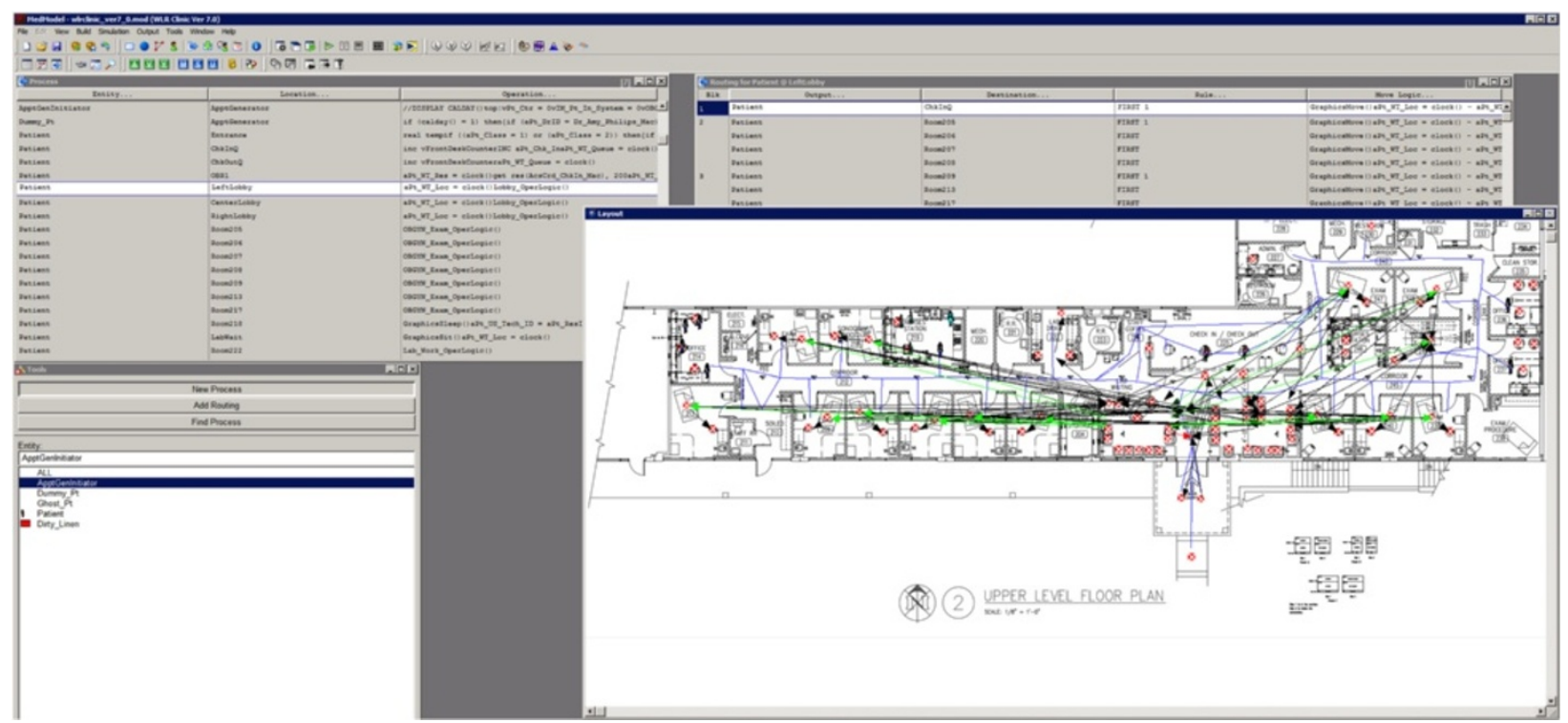

Fig. 5 Processes in MedModel 


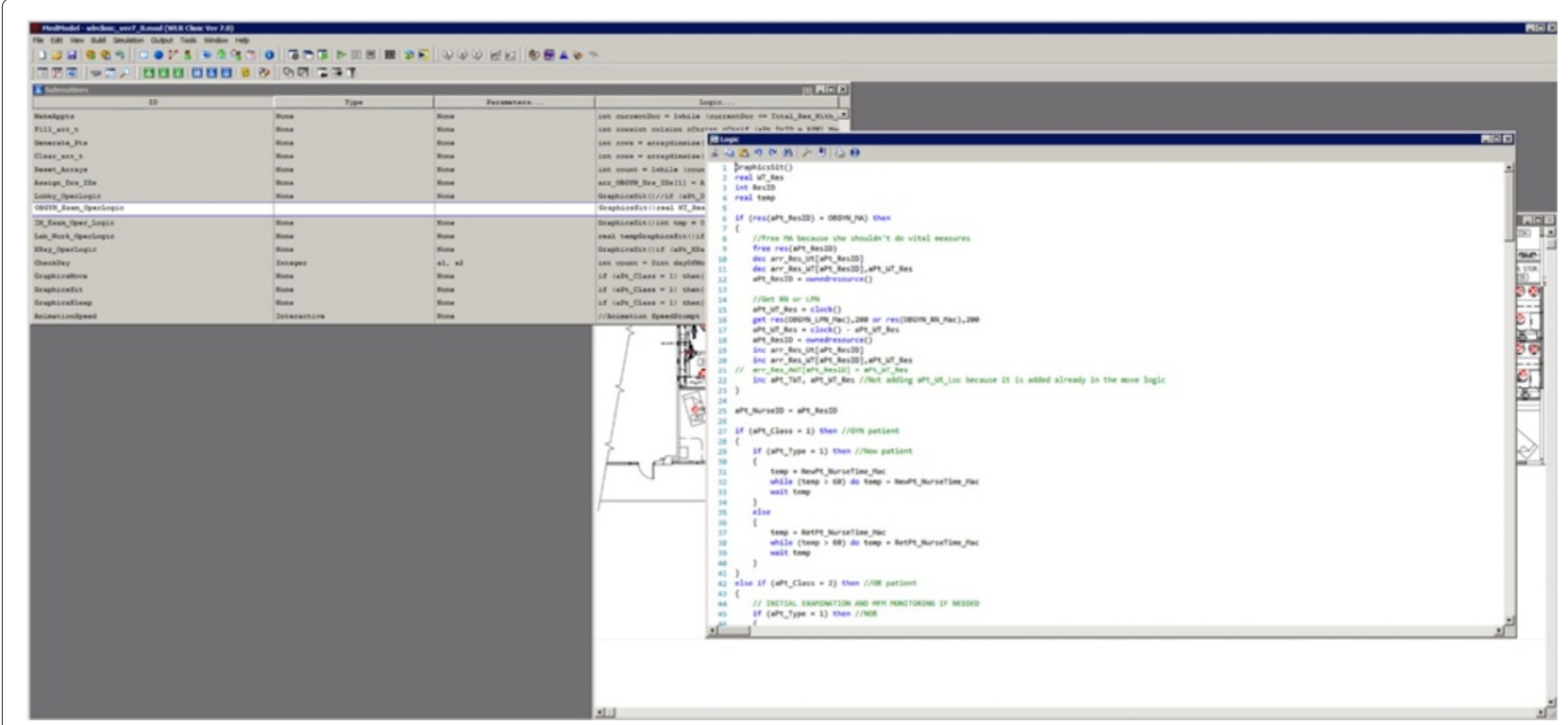

Fig. 6 Subroutines in MedModel

exact (Patient-Tracker) data to validate the developed simulation model. The validated model is then used to carry out what-if analyses. Since we measure wait times of patients at various locations $(W T)$ and their overall wait times and spent times (TWT and TST), we refer these simulation outputs as micro (for local wait times) and macro (for overall wait times and spent times) simulation analyses. We note that, in this work, the terms "micro" and "macro" are not used to represent short-term and long-term simulation durations, respectively.

\section{SimRunner}

We used SimRunner, a decision support toolbox of MedModel, to find the optimal solution of the optimization problem, which is detailed later in this paper. SimRunner takes the optimization problem as its input and runs the validated simulation model that is built using MedModel to find the optimal solution using both genetic [36] and evolutionary algorithms [37]. These algorithms help to explore specific values of contraint variables to achive the optimal solution which in turn reduces the computational complexity of the optimization algorithm.

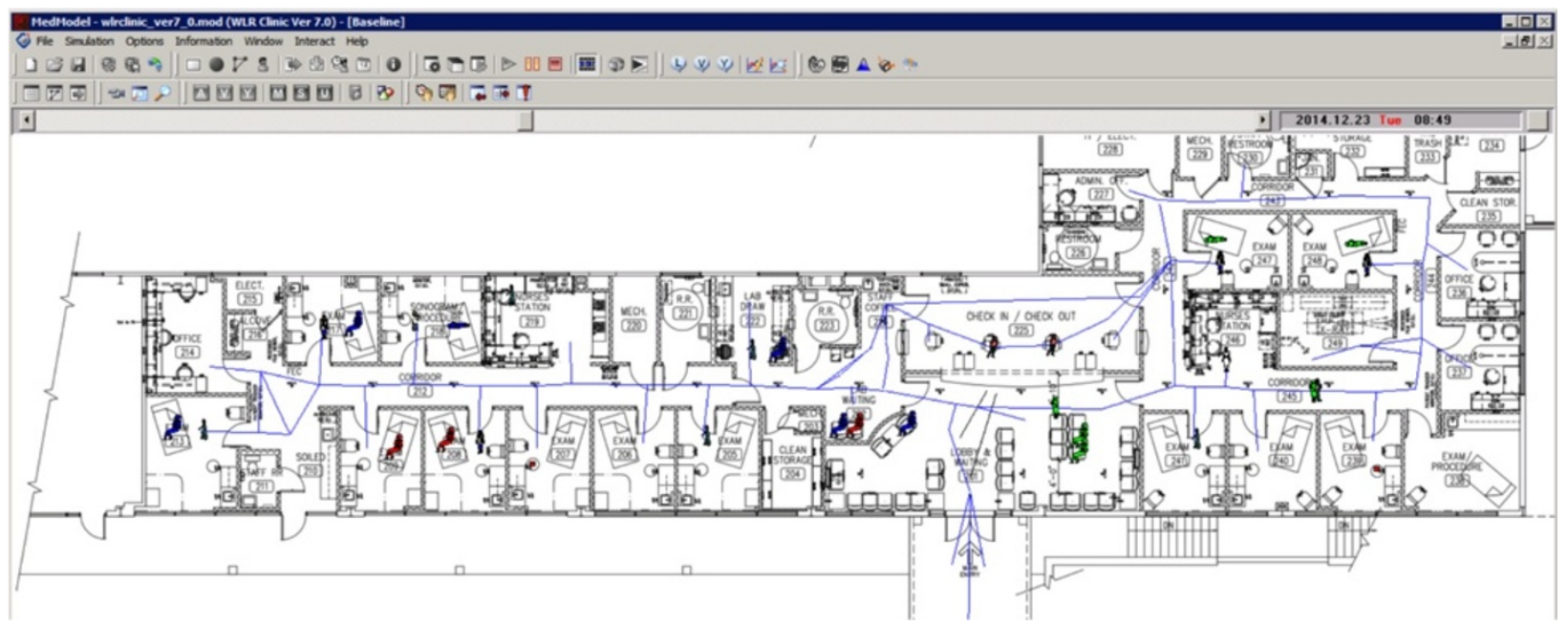

Fig. 7 Running simulation in MedModel 


\section{Notations}

Notations used for the performance measures of the clinic are tabulated in Table 5 . We note that the throughput, $\mathcal{T}$, is the total number of checked out patients by $5 \mathrm{pm}$ if the physician is either scheduled for the whole day or for the afternoon session or the total number of checked out patients by $1 \mathrm{pm}$ if the physician is scheduled for the morning session. These time limits were recommended by the clinic director.

\section{Model validation}

To validate the simulation model, the $T W T$ (see Table 5 for acronym descriptions) computed from the simulation results, after running 30 replications, were compared in Table 6 with the actual TWT extracted from the PT data. The comparison showed no statistical difference between the simulation results and actual data. Also, the comparison of WT and TST showed no statistical difference between the actual and simulation results. We used 30 replications because a sample size of 30 or more is needed to guarantee the condition that the sample mean approximately follows a normal distribution, according to the Central Limit Theorem for sample means. This condition is needed to check whether or not the difference between the actual and simulation results is statistically significant. These replications were simulated for each working day starting at 7:30 am and ending at $5 \mathrm{pm}$. The "what-if" analyses in the next section were carried out using the validated simulation model.

\section{What-if analyses}

\section{Varying number of staff}

As the physicians are the most expensive staff, we carried out "what-if" analyses by varying the number of RNs, LPNs, and MAs from 1 to 4 . Other staff were represented the same as in the validated model. We refer to the validated model as the baseline model.

\section{Modifying appointment template}

The changes to the appointment template such as reducing the number of double appointments or keeping the double appointments but moving them to different time

Table 5 Notations for the performance measures of the clinic

\begin{tabular}{ll}
\hline Acronym & Description \\
\hline$W T$ & $\begin{array}{l}\text { Average wait time of a patient for the doctor in the exam } \\
\text { room (in min) }\end{array}$ \\
TWT & $\begin{array}{l}\text { Average total wait time of a patient for the resources at } \\
\text { various locations of the clinic (in minutes) }\end{array}$ \\
TST & $\begin{array}{l}\text { Average total time of a patient from check-in to check-out } \\
\text { (in minutes) }\end{array}$ \\
$\mathcal{T}$ & $\begin{array}{l}\text { Throughput - the total number of patients seen by a } \\
\text { physician per day }\end{array}$
\end{tabular}

Table 6 Comparison of actual and simulation results

\begin{tabular}{|c|c|c|c|c|}
\hline & & \multicolumn{3}{|l|}{ TWT } \\
\hline & & Actual data & Simulation result & $p$ value \\
\hline \multirow[t]{2}{*}{ Mon } & Dr. D & 24 & 22.34 & 0.371 \\
\hline & Dr. E & 21 & 18.99 & 0.267 \\
\hline \multirow[t]{2}{*}{ Tue } & Dr. A & 35 & 37.83 & 0.539 \\
\hline & Dr. C & 33 & 34.11 & 0.761 \\
\hline \multirow[t]{3}{*}{ Thu } & Dr. A & 31 & 33.37 & 0.542 \\
\hline & Dr. B & 36 & 33.16 & 0.495 \\
\hline & Dr. D & 29 & 27.14 & 0.569 \\
\hline \multirow[t]{2}{*}{ Fri } & Dr. A & 21 & 22.14 & 0.596 \\
\hline & Dr. B & 24 & 25.37 & 0.118 \\
\hline
\end{tabular}

slots did not improve the wait time of patients. We observed that the double appointments caused more people to wait in the waiting rooms resulting in increased WT which in turn resulted in increased TWT and TST. Hence, we decided to modify the existing appointment template in Table 2 by splitting all double (two in one time slot) appointments into two single appointments in different time slots by creating two new time slots in the AM session and three slots in the PM session as shown in Table 7. This type of changes to the appointment template was found to be effective as pointed out by Ho and Lau [23]. We used this modified template to carry out "what-if" analyses to identify the optimal duration of each appointment slot by varying the duration $t_{a m}$ from $10 \mathrm{~min}$ to $16 \mathrm{~min}$ for the morning appointments and by varying the duration $t_{p m}$ from $10 \mathrm{~min}$ to $21 \mathrm{~min}$ for the afternoon appointments with the constraint that all patients are checked out by $5 \mathrm{pm}$ or earlier. The minimum value of $t_{a m}$ and $t_{p m}$ is set to be $10 \mathrm{~min}$ as per the clinic director's recommendation. The maximum value for $t_{a m}$ is set to 16 min in order to schedule all 16 patients in the morning by 12 noon whereas for $t_{p m}$, the maximum value is set to 21 min in order to schedule all 12 patients before $5 \mathrm{pm}$. We tested two different durations for morning and afternoon sessions in order to meet varying demands of these sessions which may potentially lead to overtime. This type of policies are referred as overload rules and rule delay in the literature [26].

\section{Optimization problem}

We analyzed the following optimization problem:

$$
\text { Minimize } T W T
$$

subject to the constraints

$$
\begin{aligned}
& 1 \leq x_{i} \leq 4, \quad i=1,2,3 \\
& 10 \leq t_{a m} \leq 16, \\
& 10 \leq t_{p m} \leq 21, \text { and }
\end{aligned}
$$

all patients are checked out by $5 \mathrm{pm}$ or earlier. 
Table 7 Modified appointment scheduling template

\begin{tabular}{ll}
\hline $\begin{array}{l}\text { Morning session } \\
\text { (starting at 7:45 am) }\end{array}$ & $\begin{array}{l}\text { Afternoon session } \\
\text { (starting at 12:45 pm) }\end{array}$ \\
\hline ROB & ROB \\
NGYN & NGYN \\
RGYN & RGYN \\
NOB & NOB \\
ROB & ROB \\
RGYN & ROB \\
NGYN & RGYN \\
ROB & ROB \\
NOB & ROB \\
RGYN & ROB \\
ROB & SDA \\
RGYN & SDA \\
NOB & \\
RGYN & \\
ROB & \\
ROB & \\
\hline
\end{tabular}

Here $x_{1}$ denotes the number of LPNs, $x_{2}$ denotes the number of RNs, $x_{3}$ denotes the number of MAs, and $t_{a m}$ and $t_{p m}$ denote the durations of each slot of the appointment template in Table 7 for morning and after sessions, respectively. In the constraint (2), we set the minimum number to be one for each type of nurses to make sure that at least one nurse of each type is available for the clinic model as they render different types of services.

We note that the number of nurses is independent of the slot durations. This is because the amount of time a nurse spends with a patient is obtained from the fitted probability distribution (refer Table 4) and it does not depend on the duration of the time slot. The shorter time slots lead to early arrival of patients which in turn increases the wait time of patients in different locations of the clinic due to heavy traffic, especially in the morning sessions and early afternoon sessions. Hence, it is necessary to increase the number of nurses to ease the traffic. However, on the other hand, the longer time slots lead to light traffic but less throughput. Hence, it is pertinent to identify the optimal values for the parameters in the constraints (2-4) while satisfying the constraint (5) and minimizing the objective function in (1).

The validated simulation model is modified with testing parameters based on the constraints (2) - (5). We configured SimRunner for the above optimization problem, which in turn ran the validated model for all possible parameter values in the constraints, using a sophisticated algorithm, until it found the solution that globally minimized the objective function (1). A schematic diagram of the functionality of SimRunner is shown in Fig. 8. A screenshot of an output of SimRunner is shown in Fig. 9. The optimal solution that minimized the objective function (1) is $x_{1}=2, x_{2}=1$, and $x_{3}=2, t_{a m}=16$, and $t_{p m}=19$. This means, the optimal solution is achieved by adding one more MA and keeping the morning appointments 16 min apart and afternoon appointments 19 min apart. We refer the model with these parameter values as the best model.

\section{Results}

Being a light day in terms of the number of patients scheduled, Wednesday is omitted in the "what-if" analyses. Using the optimal parameter values, we ran the simulation with 30 replications for all other working days.

\section{Wait time in the waiting room}

Figure 10 shows the average wait time of patients of all providers in the waiting room for nurses to escort them to exam rooms for the baseline scenario. The corresponding results for the best scenario are shown in Fig. 11.

\section{Utilization of staff and exam rooms}

We define the average utilization of a staff member

$=\frac{\text { total face-to-face contact time of the staff with patients per day }}{\text { total scheduled work hours of the staff per day }}$.

It does not factor in other duties of staff, such as charting and attending phone calls. Similarly, the average utilization of an exam room

$$
=\frac{\text { total time patients stayed in the exam room per day }}{\text { total scheduled work hours of the clinic per day }} \text {. }
$$

In Table 8 , the average utilizations of providers, a nurse, and an exam room are tabulated. The utilization of providers, nurses, and exam rooms throughout a typical working day of the clinic are depicted in Figs. 12, 13 and 14.

\section{Average wait times $W T, T W T, T S T$, and throughput $\mathcal{T}$}

In Tables 9 and 10, the performance measures $W T, T W T, T S T$, and $\mathcal{T}$ of the clinic corresponding to the baseline and best scenarios are compared.

The time series graphs of $W T$ and TWT of Dr. C's patients on a Tuesday are shown in Figs. 15 and 16. Similar improvements were observed across all other days for other providers and hence their graphs are omitted in the paper for brevity.

\section{Discussions}

Justification for modifying the appointment template

A patient waits in the waiting room until a nurse escorts her to an exam room if an exam room is available. Wait time of all patients of all providers in the waiting room 


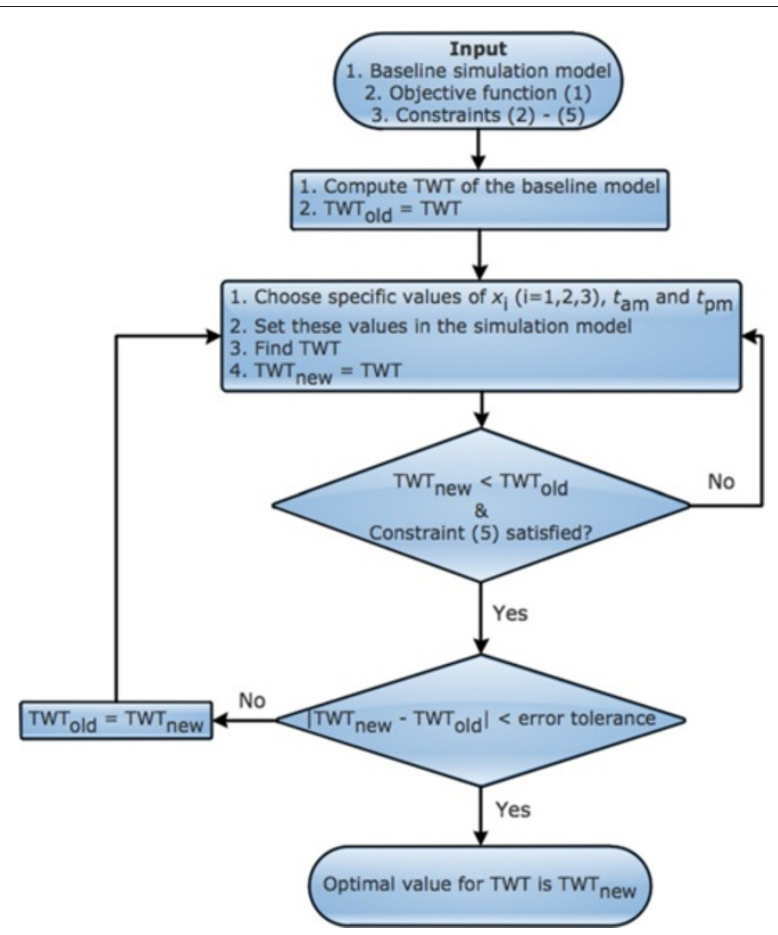

Fig. 8 Schematic diagram of functionality of SimRunner

due to unavailability of exam rooms and due to unavailability of nurses were computed in the simulation model. The average values of these wait times are plotted in Fig. 10. It is clear from Fig. 10 that throughout the day, the major delay in moving patients from the waiting room to the exam rooms is caused by the unavailability of the exam rooms rather than by the unavailability of nurses. This proves the fact that this delay cannot be avoided by adding more nurses. Since reducing the number of scheduled patients or adding more exam rooms is not cost effective, the other feasible option is to make changes to the appointment scheduling template without reducing the number of scheduled patients. Hence, we decided to include the constraints (3) and (4) in the optimization problem (1) besides modifying the appointment template by breaking all double-appointment slots into two single-appointment slots by creating new slots as shown in Table 7.

In Fig. 10, corresponding to the baseline scenario, the two major peaks in wait time occur around 12 noon and around $3 \mathrm{pm}$. The first peak is due to unavailability of all exam rooms between 11:25 am and 12 noon as no patients were moved to the exam rooms. The second peak is due to ripple effect of wait times caused by previous patients who

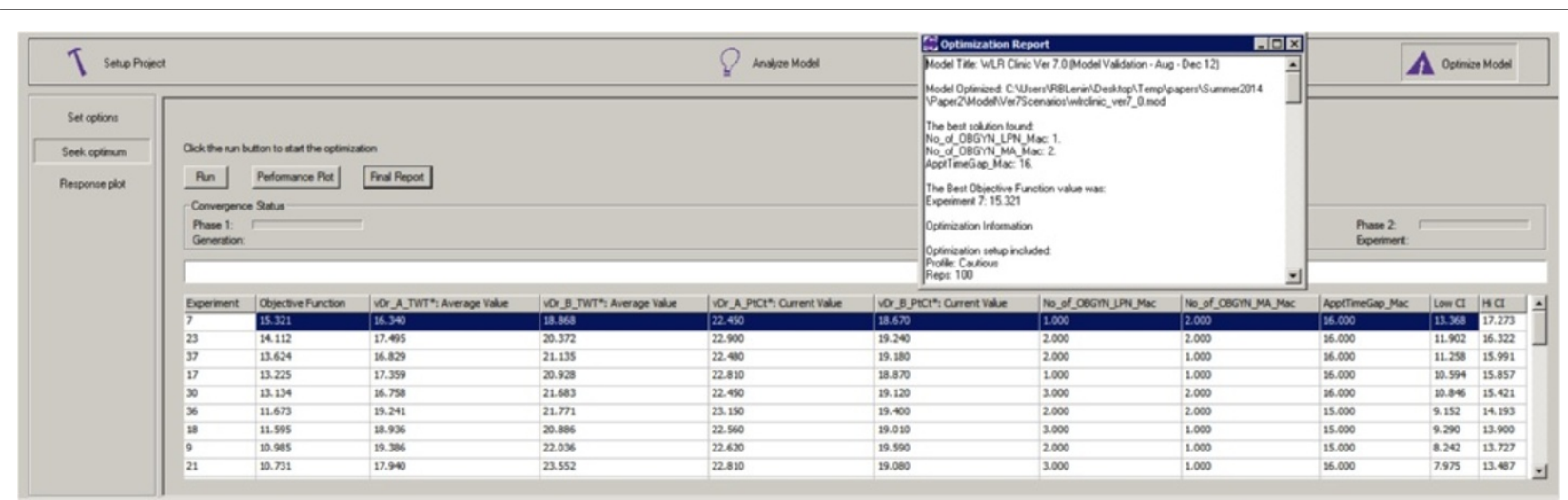

Fig. 9 Sample output of SimRunner 


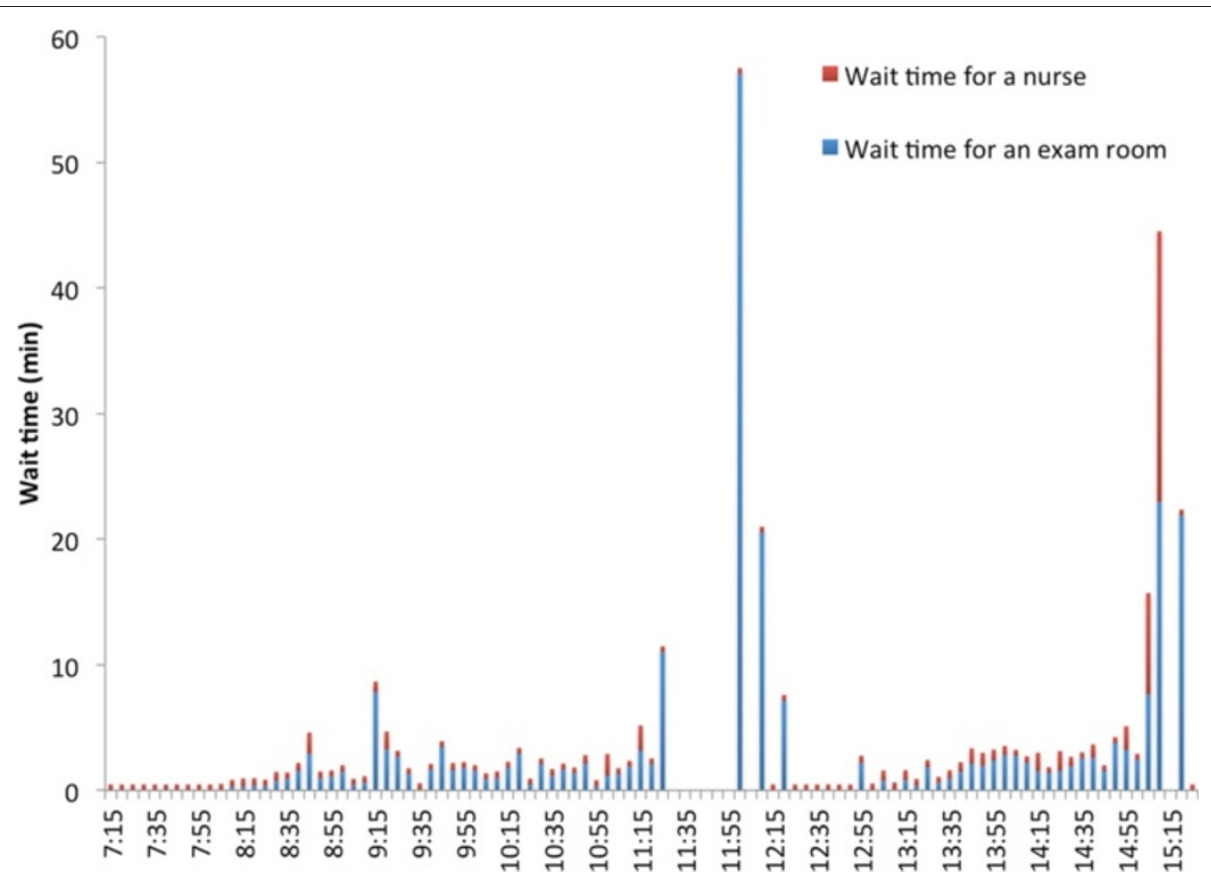

Fig. 10 Average wait time of patients in the waiting room for nurses to escort them to exam rooms in the baseline scenario

were scheduled in the early afternoon session. The second peak starts melting down as there are no more new arrival of patients after 2:45 pm.

Though the delay in moving patients to the exam rooms is still mainly due to unavailability of exam rooms in the best scenario as shown in Fig. 11, it is reduced substantially when compared to the baseline scenario (Fig. 10). In addition, the best scenario removed all major delays (e.g. more than $55 \mathrm{~min}$ around $12 \mathrm{pm}$ ) of the the baseline scenario. Moreover, between 11:30 am and $12 \mathrm{pm}$, in

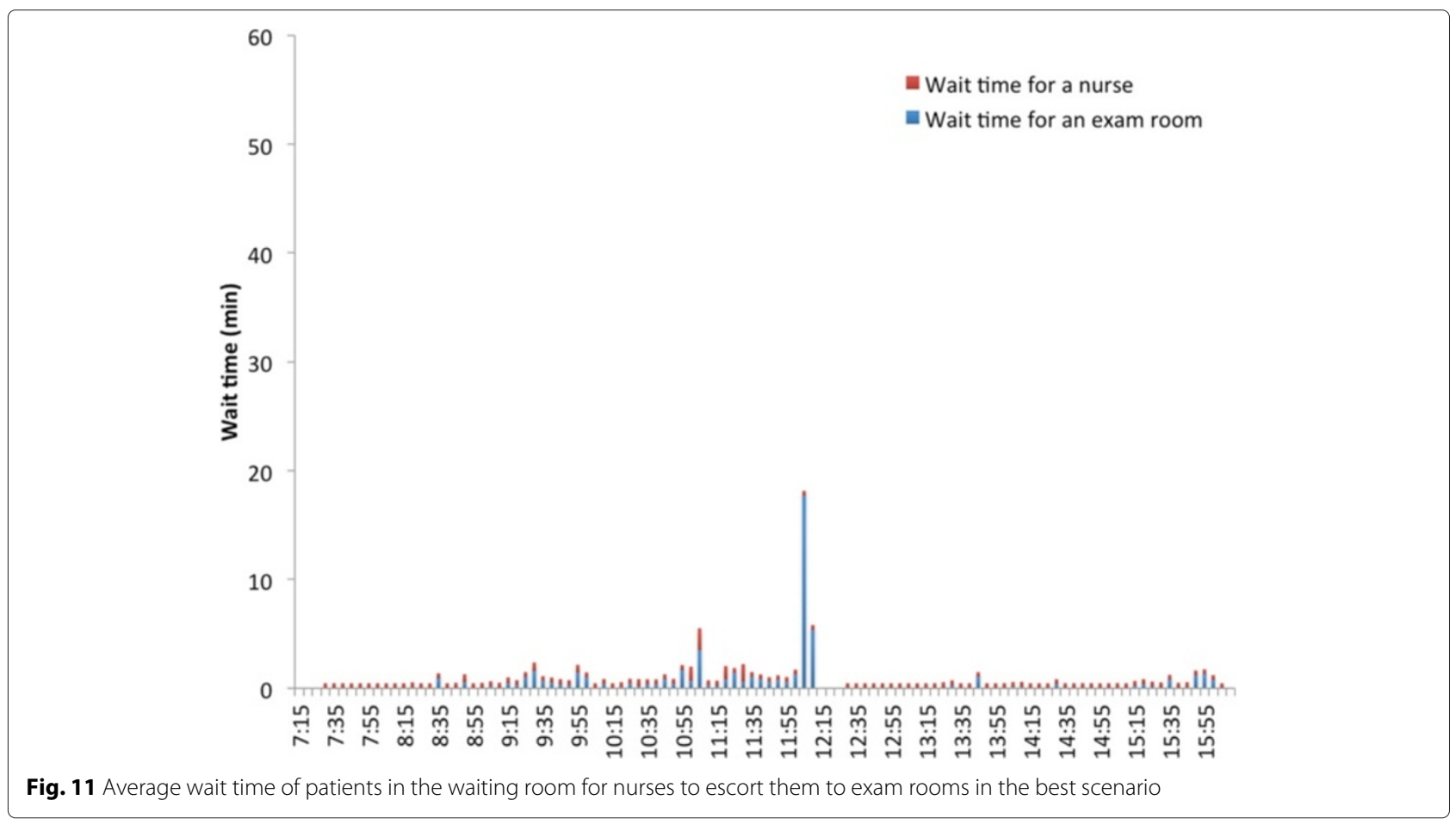


Table 8 Average utilization of staff and exam rooms

\begin{tabular}{llll}
\hline & & \multicolumn{2}{c}{ Average utilization (\%) } \\
\cline { 3 - 4 } & & Baseline & Best \\
\hline Mon & Dr. D & 72.20 & 69.71 \\
& Dr. E & 61.47 & 60.41 \\
Nurse & 34.49 & 36.46 \\
Tue & Exam room & 54.56 & 58.94 \\
& Dr. A & 69.08 & 69.17 \\
& Dr. C & 73.90 & 74.33 \\
Nhu & Nurse & 34.27 & 37.06 \\
& Exam room & 56.5 & 58.95 \\
& Dr. A & 60.50 & 51.55 \\
Dr. B & 62.16 & 57.41 \\
& Dr. D & 71.27 & 68.21 \\
& Nurse & 30.72 & 31.96 \\
Eri & Exam room & 46.33 & 47.45 \\
& Dr. A & 73.48 & 71.26 \\
& Dr. B & 74.39 & 70.68 \\
& Nurse & 32.73 & 34.33 \\
& Exam room & 57.61 & 59.4 \\
\hline
\end{tabular}

the baseline scenario, all exam rooms were occupied and no patients were moved from the waiting room to exam rooms during this time. This bottleneck is removed in the best scenario.

\section{Shift in the utilization of staff and exam rooms}

The variations in the utilization of staff and exam rooms throughout a typical working day of the clinic are captured in Figs. 12, 13 and 14. The utilization of staff and exam rooms in the best scenario is shifted to the right when compared to the baseline scenario because of the changes made to the appointment template and the morning appointments were 16 min apart and afternoon appointments were $19 \mathrm{~min}$ apart in the best scenario as identified by the optimal solution.

\section{Significant reduction in wait times}

The wait times $W T, T W T$, and TST have significantly decreased in the best scenario when compared to the baseline (Tables 9 and 10). Overall, the best scenario yielded $39.84 \%(p<.001), 30.31 \%(p<.001)$, and $15.12 \%(p<.001)$ improvement in $W T, T W T$, and TST, respectively. These improvements were achieved without compromising the utilization of staff and exam rooms (Table 8 ), and the number of patients served by $5 \mathrm{pm}$ as the $\mathcal{T}$ remains the same for both baseline and best sce-

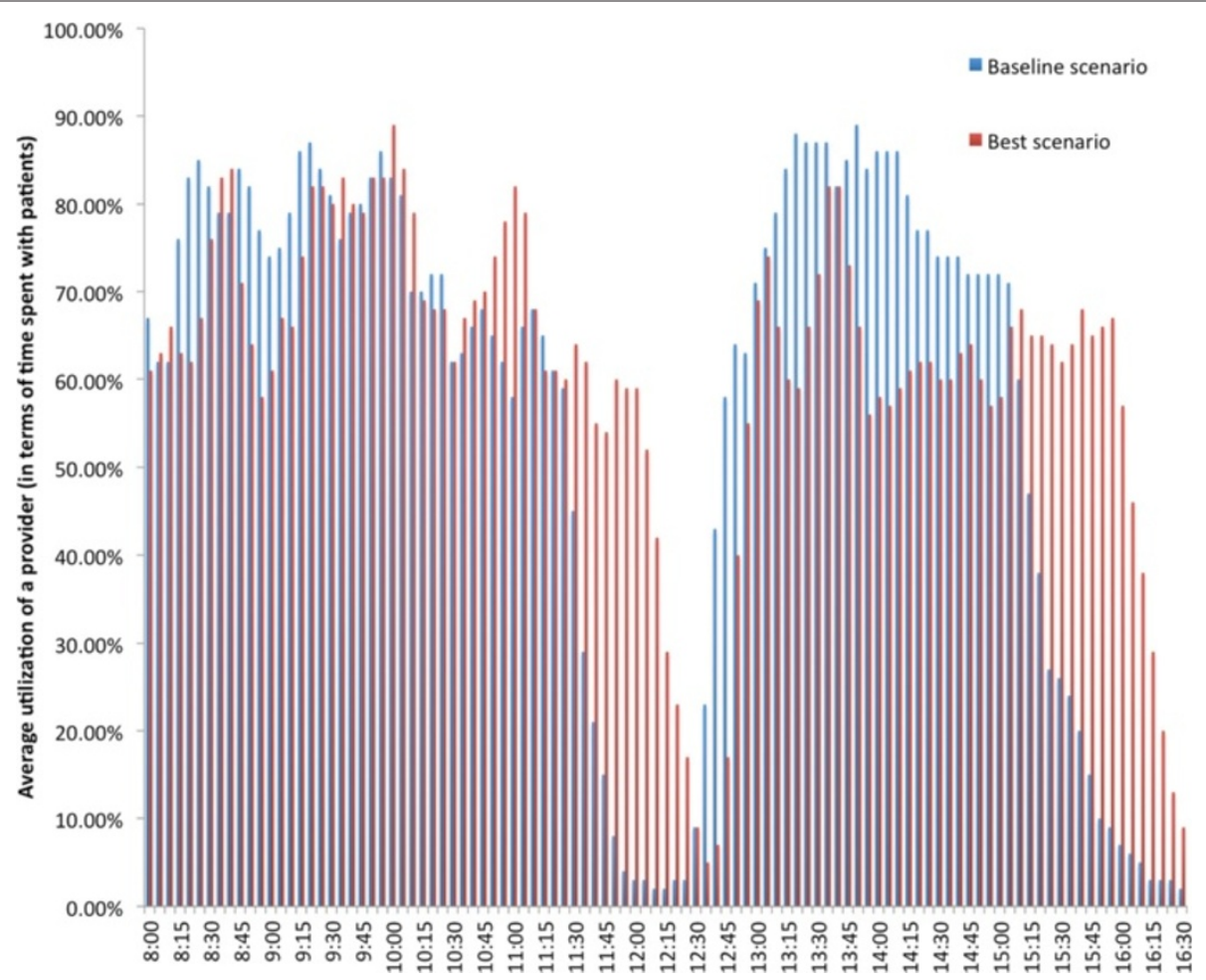

Fig. 12 Utilization of a provider 

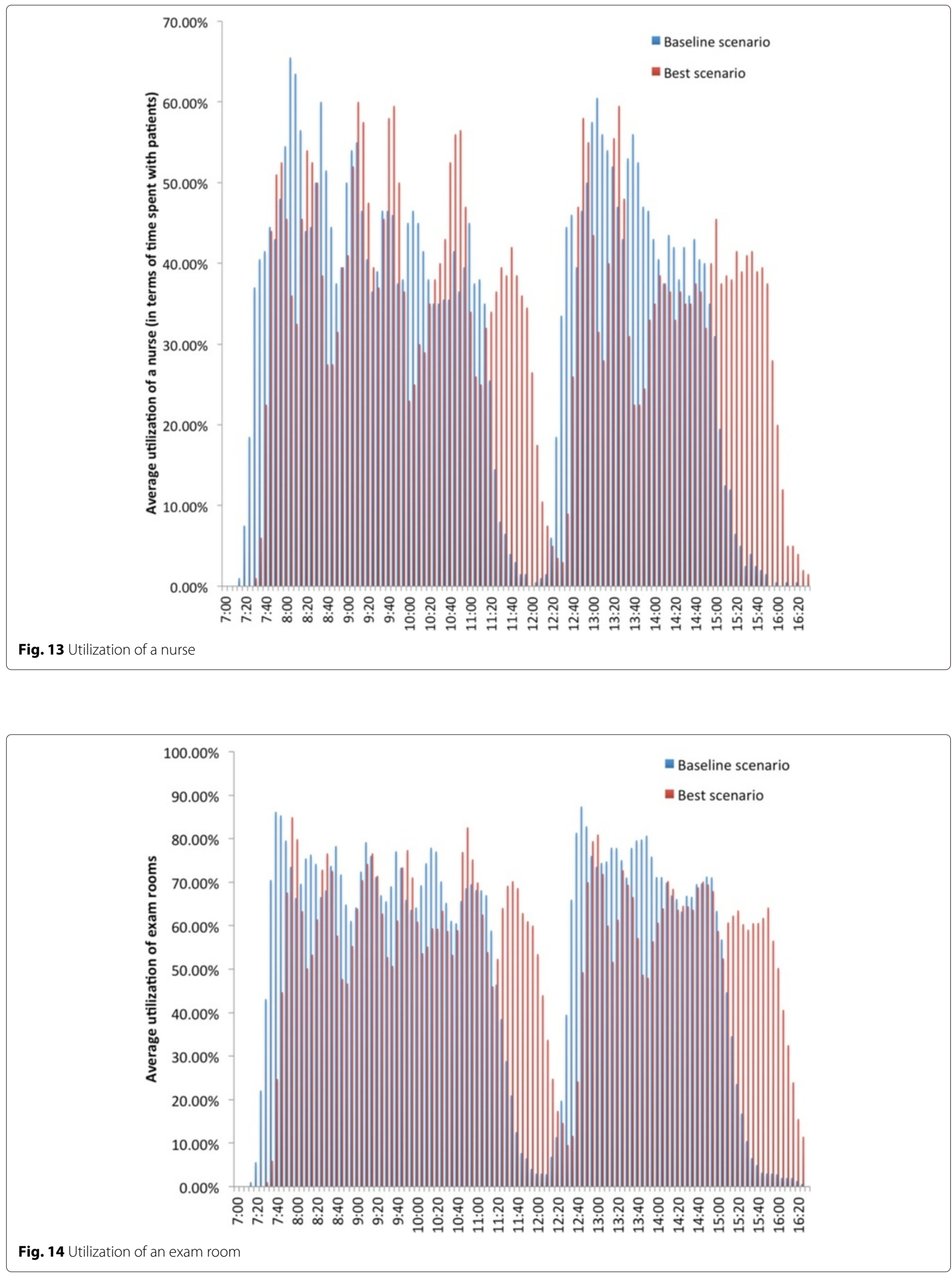
Table 9 Comparison of Baseline and Best scenarios

\begin{tabular}{|c|c|c|c|c|c|c|c|c|c|}
\hline & & \multicolumn{4}{|l|}{$W T$} & \multicolumn{4}{|l|}{ TWT } \\
\hline & & Baseline & Best & Improvement (\%) & $p$ & Baseline & Best & Improvement (\%) & $p$ \\
\hline \multirow[t]{2}{*}{ Mon } & Dr.D & 10.36 & 5.46 & 47.30 & $<.001$ & 22.34 & 14.11 & 36.84 & $<.001$ \\
\hline & Dr. E & 6.38 & 3.75 & 41.22 & $<.001$ & 18.99 & 12.42 & 34.60 & $<.001$ \\
\hline \multirow[t]{2}{*}{ Tue } & Dr. A & 9.84 & 5.92 & 39.84 & $<.001$ & 37.83 & 28.60 & 24.40 & .005 \\
\hline & Dr. C & 8.32 & 5.46 & 34.38 & $<.001$ & 34.11 & 27.07 & 20.64 & .008 \\
\hline \multirow[t]{3}{*}{ Thu } & Dr. A & 8.02 & 3.72 & 53.62 & $<.001$ & 33.37 & 17.34 & 48.04 & $<.001$ \\
\hline & Dr. B & 10.38 & 5.68 & 45.28 & .005 & 33.16 & 22.05 & 33.50 & $<.001$ \\
\hline & Dr. D & 8.31 & 5.27 & 36.58 & $<.001$ & 27.74 & 21.89 & 21.09 & .013 \\
\hline \multirow[t]{2}{*}{ Fri } & Dr. A & 11.37 & 7.96 & 29.99 & $<.001$ & 22.14 & 16.48 & 25.56 & $<.001$ \\
\hline & Dr. B & 12.32 & 8.58 & 30.36 & $<.001$ & 25.37 & 18.24 & 28.10 & $<.001$ \\
\hline Overall & & 9.48 & 5.76 & 39.84 & $<.001$ & 28.34 & 19.80 & 30.31 & $<.001$ \\
\hline
\end{tabular}

narios (Table 10). Consistent improvement in wait times is achieved in the best scenario throughout the day except at few time points (Figs. 15 and 16).

\section{Cost vs. reduction in wait time}

Since both OB/GYN and IM clinics share the same lab and MAs are the only staff handling lab works, we achieved a significant reduction in the wait times TWT and TST of patients by adding one more MA besides making changes to the appointment template. According to the U.S. Bureau of Labor Statistics [38], the median annual salary of a MA is $\$ 29,610$ which is the only additional foreseen cost to UAMS if recommended changes are made to the clinic.

\section{Conclusions}

In this paper, we: 1) used MedModel, a discrete-event simulation software, to develop a simulation model for an OB/GYN clinic, 2) used the patient tracker data to fit appropriate probability distributions for service times

Table 10 Comparison of Baseline and Best scenarios

\begin{tabular}{|c|c|c|c|c|c|c|c|}
\hline & & \multicolumn{4}{|l|}{ TST } & \multicolumn{2}{|l|}{$\mathcal{T}$} \\
\hline & & Baseline & Best & Improvement (\%) & $p$ & Baseline & Best \\
\hline \multirow[t]{2}{*}{ Mon } & Dr. D & 55.00 & 45.50 & 17.27 & $<.001$ & 24 & 24 \\
\hline & Dr. E & 51.37 & 44.20 & 13.96 & $<.001$ & 21 & 21 \\
\hline \multirow[t]{2}{*}{ Tue } & Dr. A & 72.09 & 63.80 & 11.50 & .019 & 22 & 22 \\
\hline & Dr. C & 66.66 & 59.69 & 10.46 & .015 & 24 & 24 \\
\hline \multirow[t]{3}{*}{ Thu } & Dr. A & 65.61 & 50.10 & 23.64 & $<.001$ & 9 & 9 \\
\hline & Dr. B & 69.85 & 57.04 & 18.34 & $<.001$ & 16 & 16 \\
\hline & Dr. D & 59.59 & 52.98 & 11.09 & $<.001$ & 14 & 14 \\
\hline \multirow[t]{2}{*}{ Fri } & Dr. A & 55.80 & 46.30 & 17.03 & $<.001$ & 23 & 23 \\
\hline & Dr. B & 62.95 & 54.90 & 12.79 & $<.001$ & 19 & 19 \\
\hline Overall & & 62.10 & 52.72 & 15.12 & $<.001$ & & \\
\hline
\end{tabular}

of staff and to validate the model, and 3) carried out "what-if" analyses using the validated model to find the optimal solution of an optimization problem of minimizing the average wait times of patients in the clinic subject to the constraints of varying number of nurses and varying time between appointments of a modified appointment template. The existing appointment template was modified by breaking all double-appointment slots into two single-appointment slots by creating new slots.

The optimal solution was achieved by adding one more MA and keeping the morning appointments 16 min apart and afternoon appointments 19 min apart. Besides removing all peak wait times and bottlenecks around noon and late in the afternoon, the changes suggested by the optimal solution led to a significant reduction in the wait time of patients without compromising the utilization of the staff and exam rooms or throughput of patients. We achieved these improvements without affecting the actual clinic activities which the clinic management had difficulty achieving with many manual interventions. Moreover, these manual interventions did not reveal the fact that the unavailability of exam rooms was the main cause for the major delay in moving the patients from the waiting room to exam rooms. An extensive list of tables and figures were provided to describe the clinic operations, to describe the simulation tools, and to illustrate the improvements of all micro and macro performance measures of the clinic.

It has been observed that, on average, providers are occupied for at least $15 \mathrm{~min}$ more between 12:30 pm and $1 \mathrm{pm}$ in the best scenario when compared to the baseline (Figs. 14 and 15) due to more appointment slots with 16 and 19 min apart for morning and after sessions, respectively. Similar, observation was made for nurses. 


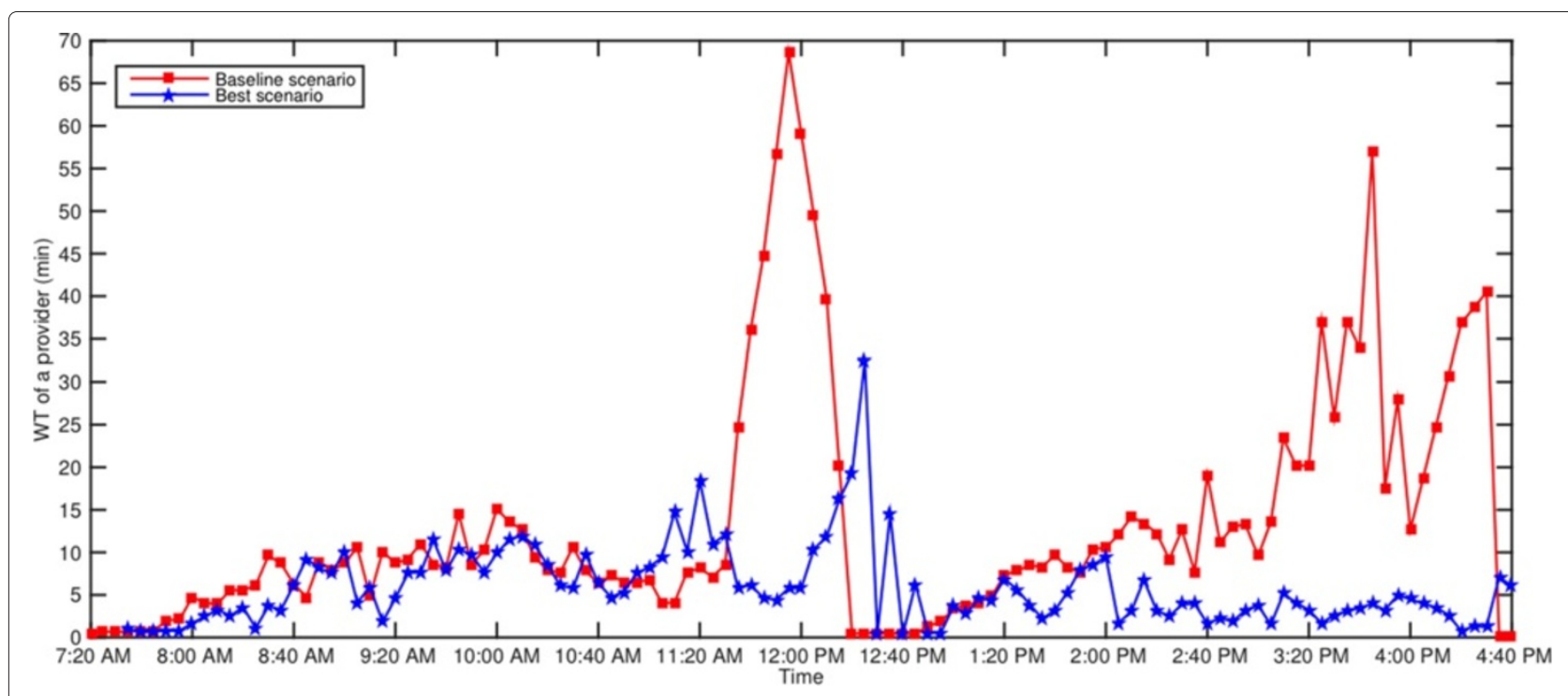

Fig. 15 WT of Dr. C's patients on a Tuesday

This type of changes to the appointment template which lead to overtime of staff is referred as overload rules and rule delay in the literature. As part of the future plan, after consulting with the clinic management, staggered working schedules for nurses and providers will be proposed to address the overtime of staff.

The future plan is also to extend this model for the other two UAMS OB/GYN outpatient clinics to reduce patient wait times by identifying optimal number of resources for these clinics.
Competing interests

The authors declare that they have no competing interests.

\section{Authors' contributions}

RBL developed the simulation model, carried out analysis, and drafted the analysis section. CLL helped in the sequence alignment and design of the study, and provided interpretation of results. WCH drafted the abstract and background sections and helped in interpreting results. NAM helped in providing clinic description, design of the study and interpretation of results. PL performed the statistical analysis. HE participated in acquisition and interpretation of data and results, and helped to draft the manuscript. All authors read and approved the final manuscript.

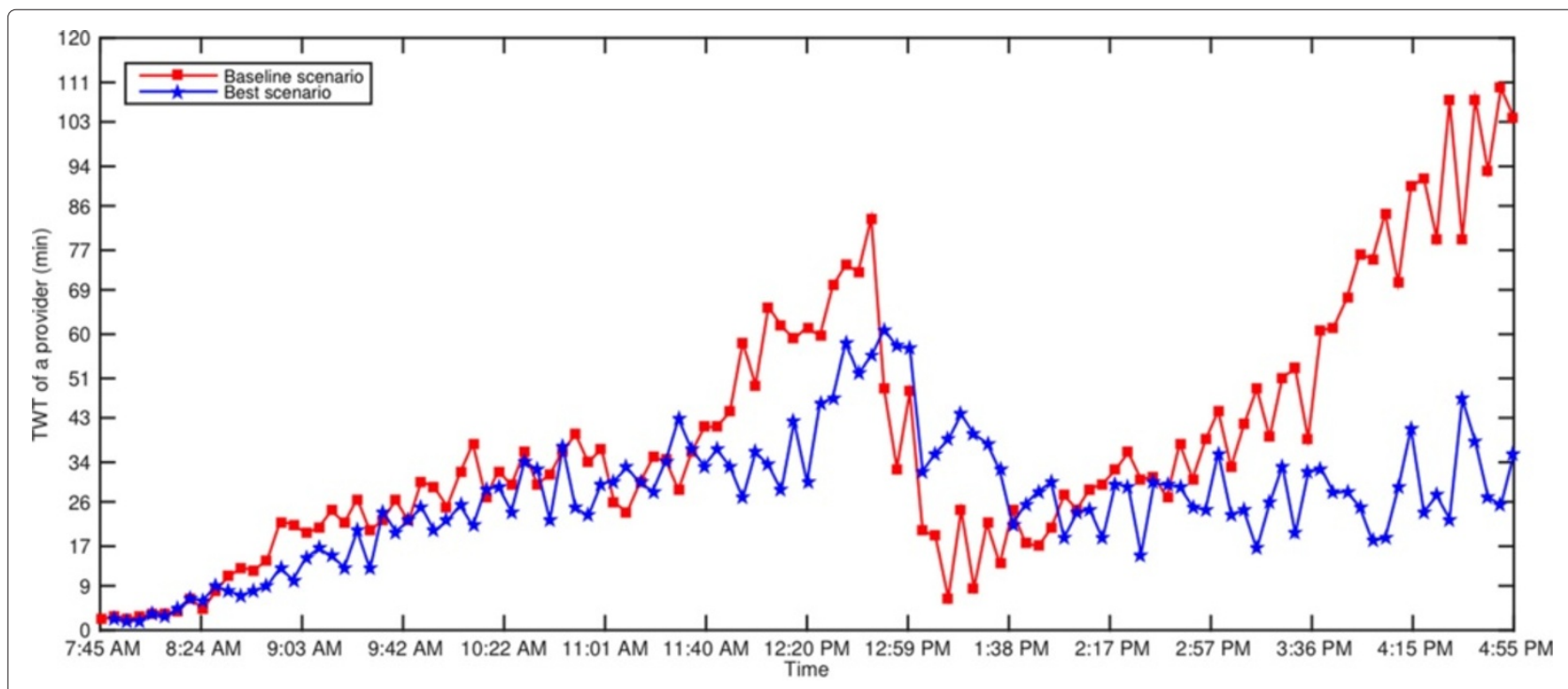

Fig. 16 TWT of Dr. C's patients on a Tuesday 


\section{Acknowledgements}

The authors thank the anonymous reviewers for detailed comments and suggestions which improved the quality of the paper.

\section{Author details}

${ }^{1}$ Department of Mathematics, University of Central Arkansas, 201 Donaghey Avenue, 72035 Conway, Arkansas, USA. ${ }^{2}$ Department of OB/GYN, University of Arkansas for Medical Sciences, 4301 West Markham Street, 72205 Little Rock, Arkansas, USA. ${ }^{3}$ College of Medicine, University of Arkansas for Medica Sciences, 4301 West Markham Street, 72205 Little Rock, Arkansas, USA.

\section{Received: 5 August 2014 Accepted: 17 August 2015}

\section{Published online: 16 September 2015}

\section{References}

1. McCarthy K, McGee HM, O'Boyle CA. Outpatient clinic waiting times and non-attendance as indicators of quality. Psychol Health Med. 2000;5(3): 287-93.

2. Hill CJ, Joonas K. The impact of unacceptable wait time on healthcare patients' attitudes and actions. Health Mark Quartely. 2005;23(2):69-87.

3. Gourdji I, McVey L, Loiselle C. Patients' satisfaction and importance ratings of quality in an outpatient oncology center. J Nurse Care Qual. 2003;18(1):43-55.

4. Hart M. Improving out-patient clinic waiting times: Methodological and substantive issues. Int J Health Care Qual Assur. 1995;8(6):14-22.

5. Eilers GM. Improving patient satisfaction with waiting time. J Am Coll Health. 2004;53(1):41-8.

6. Anderson RT, Camacho FT, Balkrishnan R. Willing to wait? The influence of patient wait time on satisfaction with primary care. BMC Health Serv Res. 2007;7(31):1-5.

7. Schoenfelder T, Schaal T, Klewer J, Kugler J. Patient satisfaction and willingness to return to the provider among women undergoing gynecological surgery. Arch Gynecol Obstet. 2014;290(4):683-90. doi:10.1007/s00404-014-3248-y.

8. Zoller JS, Lackland DT, Silverstein MD. Predicting patient intent to return from satisfaction scores. J Ambul Care Manag. 2001;24(1):44-50.

9. Brailsford SC, Harper PR, Patel B, Pitt M. An analysis of the academic literature on simulation and modelling in health care. J Simul. 2009;3: 130-40.

10. Jun GT, Morris Z, Eldabi T, Harper P, Naseer A, Patel B, et al. Development of modelling method selection tool for health services management: From problem structuring methods to modelling and simulation methods. BMC Health Serv Res. 2011;11(108):1-11.

11. Lee LH, Chew EP, Frazier PI, Jia QS, Chen CH. Advances in simulation optimization and its applications. IIE Trans. 2013;45(7):683-4.

12. Harper PR, Gamlin HM. Reduced outpatient waiting times with improved appointment scheduling: a simulation modelling approach. OR Spectr. 2003;25(2):207-22.

13. Jacobson SH, Hall SN, Swisher JR. Discrete-event simulation of health care systems In: Hall RW, editor. Patient flow: Reducing delay in healthcare delivery vol. 91. Springer-Verlag, USA: Springer; 2006. p. 211-52. Chap. 8

14. Chand S, Moskowitz H, Norris JB, Shade S, Willis DR. Improving patient flow at an outpatient clinic: study of sources of variability and improvement factors. Health Care Manag Sci. 2009;12(3):325-40.

15. Rohleder TR, Lewkonia P, Bischak DP, Duffy P, Hendijani R. Using simulation modeling to improve patient flow at an outpatient orthopedic clinic. Health Care Manag Sci. 2011;14(2):135-45.

16. van Sambeek JRC, Cornelissen FA, Bakker PJM, Krabbendam JJ. Models as instruments for optimizing hospital processes: a systematic review. Int J Health Care Qual Assur. 2010;23(4):356-77.

17. Banks J, Carson JS, Nelson BL, Nicol DM. Discrete-Event System Simulation, 5th ed. USA: Prentice Hall; 2010.

18. Denton B, Gupta D. A sequential bounding approach for optimal appointment scheduling. IEEE Trans. 2003:35(11):1003-16.

19. Robinson LW, Chen RR. Scheduling doctors' appointments: Optimal and empirically-based heuristic policies. IIE Trans. 2003;35(3):295-307.

20. Muthuraman K, Lawley M. A stochastic overbooking model for outpatient clinical scheduling with no-shows. IIE Trans. 2008;40(9):820-37.

21. Huang YL, Hancock WM, Herrin GD. An alternative outpatient scheduling system: Improving the outpatient experience. IIE Trans Healthc Syst Eng. 2012;2(2):97-111.
22. CoxTF, Birchall JP, Wong H. Optimising the queuing system for an ear, nose and throat outpatient clinic. J Appl Stat. 1985;12(2):113-26.

23. Ho CJ, Lau HS. Minimizing total cost in scheduling outpatient appointments. Manag Sci. 1992;38(12):1750-64.

24. Gupta D, Denton B. Appointment scheduling in health care: Challenges and opportunities. IIE Trans. 2008:40(8):800-19.

25. Yang KK, Lau ML, Quek SA. A new appointment rule for a single-server, multiple-customer service system. Nav Res Logist. 1998;45(3):313-26.

26. Rohleder TR, Klassen KJ. Rolling horizon appointment scheduling: A simulation study. Health Care Manag Sci. 2002;5(3):201-9.

27. Swisher JR, Jacobson SH. Evaluating the design of a family practice healthcare clinic using discrete-event simulation. Health Care Manag Sci. 2002;5(2):75-88.

28. Rohleder TR, Bischak DP, Baskin LB. Modeling patient service centers with simulation and system dynamics. Health Care Manag Sci. 2007;10(1):1-12.

29. Santibáñez P, Chow VS, French J, Puterman ML, Tyldesley S. Reducing patient wait times and improving resource utilization at british columbia cancer agency's ambulatory care unit through simulation. Health Care Manag Sci. 2009:12(4):392-407.

30. White DL, Froehle CM, Klassen KJ. The effect of integrated scheduling and capacity policies on clinical efficiency. Prod Oper Manag. 2011;20(3) 442-55.

31. Carlson RC, Hershey JC, Kropp DH. Use of optimization and simulation models to analyze outpatient health care settings. Decis Sci. 1979;10(3): 412-33.

32. Klassen KJ, Yoogalingam R. Improving performance in outpatient appointment services with a simulation optimization approach. Prod Oper Manag. 2009;18(4):447-58.

33. Ahmed MA, Alkhamis TM. Simulation optimization for an emergency department healthcare unit in kuwait. Eur J Oper Res. 2009;198(3):936-42.

34. Stat:Fit: Statistically fit software. 2014. http://www.geerms.com.

35. MedModel 2014. The industry standard for healthcare simulations. 2014 http://www.promodel.com.

36. Haupt RL, Haupt SE. Practical Genetic Algorithms, 2nd ed. New York, NY Wiley; 2004

37. Bowden R, Bullington SF. An evolutionary algorithm for discovering manufacturing control strategies. Evolutionary Algorithms in Management Applications, vol. Part Two. Berlin Heidelberg: Springer; 1995, pp. 124-38.

38. US Bureau of Labor Statistics. 2013. Retrieved from http://data.bls.gov/oes.

\section{Submit your next manuscript to BioMed Central and take full advantage of:}

- Convenient online submission

- Thorough peer review

- No space constraints or color figure charges

- Immediate publication on acceptance

- Inclusion in PubMed, CAS, Scopus and Google Scholar

- Research which is freely available for redistribution 